\title{
Development of Micro Wide Angle Fovea Lens
}

\section{- Lens Design and Production of Prototype-_}

\author{
Sota Shimizu*a) Member, Takumi Hashizume** Non-member
}

(Manuscript received May 7, 2012, revised Sep. 4, 2012)

\begin{abstract}
This paper describes the development of a biologically inspired wide-angle fovea (WAF) lens well known as a significant part of a biomimetic vision sensor. The authors develop a micro WAF lens suitable for a board lens camera with a $1 / 3$ inch imaging chip. The prototype of this Micro WAF lens has a smaller diameter and length, i.e., $\phi 8 \mathrm{~mm}$ $\times 15 \mathrm{~mm}$, than half of those of the WAF lens that the author produced formerly, i.e., $\phi 16 \mathrm{~mm} \times 32 \mathrm{~mm}$. However, its central field of view (FOV), called herein fovea, has a much higher resolution; that is, its projected image is more highly distorted than that projected by the former WAF lens. The central FOV is approximately $10 \%$ of the entire FOV within an incident angle of $2.5^{\circ}$ in Micro WAF lens, whereas it is approximately $10 \%$ within an incident angle of $10^{\circ}$ in the former WAF lens. We mainly focus on determining its specification as the first step of a lens design procedure and discussing the results simulated using the lens design software.
\end{abstract}

Keywords: fovea sensor, wide-angle lens, micro wide-angle fovea lens, board camera, lens design

\section{Introduction}

The human eye has a 120-degree wide field of view (FOV) in the horizontal direction and its visual acuity is the highest in the central FOV and decreases rapidly towards the peripheral FOV, i.e., it has an explicit attention area in its FOV ${ }^{(1)}$. The wide-angle fovea (WAF) vision sensor, biologicallyinspired from the well-known distribution of the human visual acuity mentioned in the above, is quite significant as a multi-purpose sensor just at present particularly when it is applied for information communication by a PC internet, a mobile network and etc., because it can acquire information from an environment as in detail as possible with as small data amount as possible by controlling its view direction. We often hear technical terms such as a communication speed in internet and a packet fee in mobile network. Concretely, the WAF sensor designed in this paper can reduce a cost of the packet fee drastically into approximately $1 / 160$ so that it solves a traditional bottle-neck problem of data transmission. Guess the WAF sensor motorized for changing its view direction is applicable for a security camera system installed in a 24-hour supermarket. This security system needs to record and keep a long-time movie (a couple of months, often). In this situation, a realistic data storage problem causes to reduce an image quality of the movie (as gray-scale and quite a low resolution). Utilizing the WAF sensor for this kind of applications, we can improve the image quality without increasing the strain on the data storage.

\footnotetext{
a) Correspondence to: Sota Shimizu. E-mail: sota@cvl.iis. u-tokyo.ac.jp

* The University of Tokyo

4-6-1, Komaba, Meguro-ku, Tokyo 153-8505, Japan

** Waseda University

17, Kikui-cho, Shinjuku-ku, Tokyo 162-0044, Japan
}

This paper describes development of a new wide-angle fovea (WAF) lens, which plays the major role in the fovea sensor system. We focus on minimizing its size and enhancing its functionality in order to spread its application fields more broadly. Section 2 surveys related works and compare the WAF lens the author produced formerly ${ }^{(2)}$ with the WAF sensor system realized by other methods. Section 3 introduces the Advanced Wide Angle Foveated (AdWAF) model, determines a target specification taking into account minimizing and functionalizing a newly-developed WAF lens (we call this new lens Micro WAF lens hereafter), and discusses its resolution and optical performance based on a CAD simulation data of Micro WAF lens. Section 4 summarizes this paper describing findings from results and mentions future works.

\section{Related Works}

The former WAF lens is used by attaching with a commercially-available C-mount industrial camera having an array-structure vision chip with uniform spatial resolution, such as CCD and CMOS cameras ${ }^{(2)}$. Magnification of the WAF lens is the highest in the optical axis direction and decreases rapidly as an incident angle gets larger, i.e., a projected image of the WAF lens is highly-distorted. A lens type of the WAF sensor system, comprising such a specialmade wide-angle lens and a commercially-available area sensor, acquires a WAF image with space-variant resolution by distorting the projected image highly.

The log-polar mapping, inspired from biological studies on a brain-vision system of primates, is often applied for computer vision as another method by which we realizes the WAF image. Some acquire the WAF image by software downsampling from the uniform-resolution image as a united size of pixels gets larger towards its peripheral $\mathrm{FOV}^{(3)}$, and other 
do it by using a special-made CCD or CMOS vision chip, where photosensitive elements are arranged uniformly in the central FOV and their size increases towards the peripheral FOV according to an exponential function ${ }^{(6)-(8)}$. This specialmade vision chip type combines a commercially-available conventional wide-angle lens, while the special-made lens type combines a commercially-available conventional vision chip. The latter type is more suitable for achieving a complicated image model based on combination of multiple coordinate systems than the former. In addition, the latter type realizes higher spatial resolution in its central FOV than the former if a density of the photosensitive elements used in both types is the same, because the latter optically magnifies its central FOV more largely.

The log-polar mapping not only reduces an amount of image information drastically but also gives us rotation- and scale-invariant property which is useful for pattern matching, i.e., we can extract a unique feature from the log-polar image of a target even if the target is rotated, magnified and demagnified ${ }^{(5)}$. The WAF lens produced by Kuniyoshi and et al. (K lens hereafter) has a camera model including a logarithmic function as a part of its spherical projection taking into account this property ${ }^{(3)}$. In other words, the $\mathrm{K}$ lens acquires the WAF image by connecting a linear planar projection and a linear spherical projection using the logarithmic spherical projection. Thus, an image by the $\mathrm{K}$ lens has a logarithmic spherical part having a rotation- and scale-invariant property. It is consistent to use a logarithmic function in order to acquire the WAF image. But the scale-invariant property of the $\mathrm{K}$ lens is just approximation to a target on a plane. This means that the feature is no longer unique because of an approximation error if incident angles corresponding to boundaries of the logarithmic spherical part are large to some degree. Paying attention to this problem, the author designed and proposed the Advanced Wide Angle Foveated (AdWAF) model which gives us a complete scale-invariant property to a target on a plane by having both logarithmic planar part and logarithmic spherical part ${ }^{(9)-(12)}$.

\section{Design of Micro WAF Lens}

\subsection{Advanced Wide Angle Foveated Model}

Figure 1 compares input images by the former WAF lens and by the pinhole camera (PHC) lens. Assume these input images have the same FOV, i.e., the same 120-degree visual angle and the same number of pixels. This former WAF lens realizes about 120-degree wide FOV and adequate high

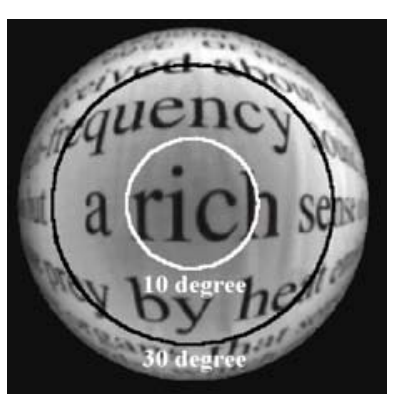

(a) Input image by the former WAF lens

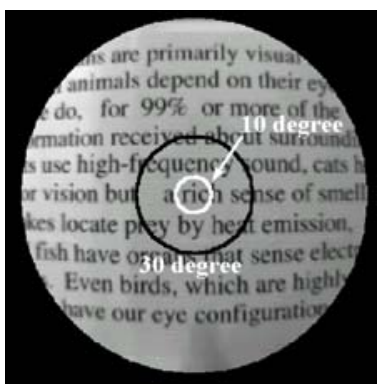

(b) PHC lens image
Fig. 1. Comparing the former WAF lens and PHC lens image resolution in the central FOV, simultaneously, by distorting its projected image intentionally as mentioned in Section II. The author introduces the Advanced Wide Angle Foveated (AdWAF) model in order to use the WAF image more efficiently ${ }^{(10)}$. Figure 2 shows a camera model in which a planar projection and a spherical projection are combined. The former is a perspective projection which is linear to a tangent of an incident angle, $\theta$, to the lens optical center. The latter is a projection which is linear to the incident angle $\theta$. The projection of this camera model is expressed as follows:

$$
\begin{aligned}
& p=f_{1} \tan \theta \quad\left(0 \leq \theta \leq \theta_{1}\right), \cdots \ldots \ldots \ldots \ldots \ldots \ldots \\
& p=f_{2}\left(\theta-\theta_{1}\right)+f_{1} \tan \theta_{1} \quad\left(\theta_{1} \leq \theta \leq \theta_{\max }\right), \cdots \cdots
\end{aligned}
$$

where $f_{1}$ and $f_{2}$ are focal lengths to a projected plane and a projected spherical surface, respectively.

The AdWAF model is defined based on combining linear coordinates and logarithmic coordinates with 2 types of projections by eqs. (1) and (2).

$$
\begin{aligned}
& r=r_{\max } c_{0} f_{1} \tan \theta \quad\left(0 \leq \theta \leq \theta_{0}\right), \cdots \cdots \cdots \cdots \cdots \\
& r=r_{\max }\left\{c_{1} \log _{a}\left(f_{1} \tan \theta\right)+d_{1}\right\} \quad\left(\theta_{0} \leq \theta \leq \theta_{1}\right), \cdots \\
& r=r_{\max }\left\{c_{2} \log _{b}\left(f_{2} \theta\right)+d_{2}\right\} \quad\left(\theta_{1} \leq \theta \leq \theta_{2}\right), \cdots \cdots \\
& r=r_{\max }\left(c_{3} f_{2} \theta+d_{3}\right) \quad\left(\theta_{2} \leq \theta \leq \theta_{\max }\right), \cdots \cdots \cdots \cdots
\end{aligned}
$$

where $r$ is an image height corresponding to the incident angle $\theta, r_{\max }$ is the maximum image height when $\theta=\theta_{\max }, c_{i}$ $(i=0,1,2,3)$ is a scale modification factor to control an image size of each region. $d_{i}(i=0,1,2)$ is expressed as follows:

$$
\begin{aligned}
& d_{1}=c_{0} f_{1} \tan \theta_{0}-c_{1} \log _{a}\left(f_{1} \tan \theta_{0}\right), \ldots \ldots \ldots \ldots \ldots \\
& d_{2}=c_{1} \log _{a}\left(f_{1} \tan \theta_{1}\right)-c_{2} \log _{b}\left(f_{2} \theta_{1}\right)+d_{1}, \ldots \ldots \ldots \\
& d_{3}=c_{2} \log _{b}\left(f_{2} \theta_{2}\right)-c_{3} f_{2} \theta_{2}+d_{2} . \ldots \ldots \ldots \ldots \ldots
\end{aligned}
$$

Equations (3) to (6) are continuous. In addition, assuming that their first derivations of these equations are continuous, the following equations are derived.

$$
\begin{aligned}
& f_{1}=\frac{1}{\tan \theta_{0}} /\left\{1+\log \frac{\tan \theta_{1}}{\tan \theta_{0}}\right. \\
& \left.+\frac{\theta_{1}}{\cos \theta_{1} \sin \theta_{1}}\left(\frac{\theta_{\max }-\theta_{2}}{\theta_{2}}+\log \frac{\theta_{2}}{\theta_{1}}\right)\right\}, \cdots \\
& f_{2}=\frac{f_{1} \tan \theta_{0}}{\cos \theta_{1} \sin \theta_{1}} \cdot \frac{\theta_{1}}{\theta_{2}}, \ldots \ldots \ldots \ldots \ldots \ldots \ldots \\
& a=\exp \left(\frac{1}{f_{1} \tan \theta_{0}}\right) \text {, } \\
& b=\exp \left(\frac{1}{f_{2} \theta_{2}}\right) \text {. }
\end{aligned}
$$

The AdWAF model divides its FOV into 4 regions, i.e., fovea $\left(0 \leq \theta \leq \theta_{0}\right)$, para-fovea $\left(\theta_{0} \leq \theta \leq \theta_{1}\right)$, near-periphery $\left(\theta_{1} \leq \theta \leq \theta_{2}\right)$, and periphery $\left(\theta_{2} \leq \theta \leq \theta_{\max }\right)$. The fovea is planar and its image height is linear to the object height $h$. On the other hand, the periphery is spherical and its image height is linear to the incident angle $\theta$. Figure 3 simulates images remapped by the AdWAF model and the PHC lens 


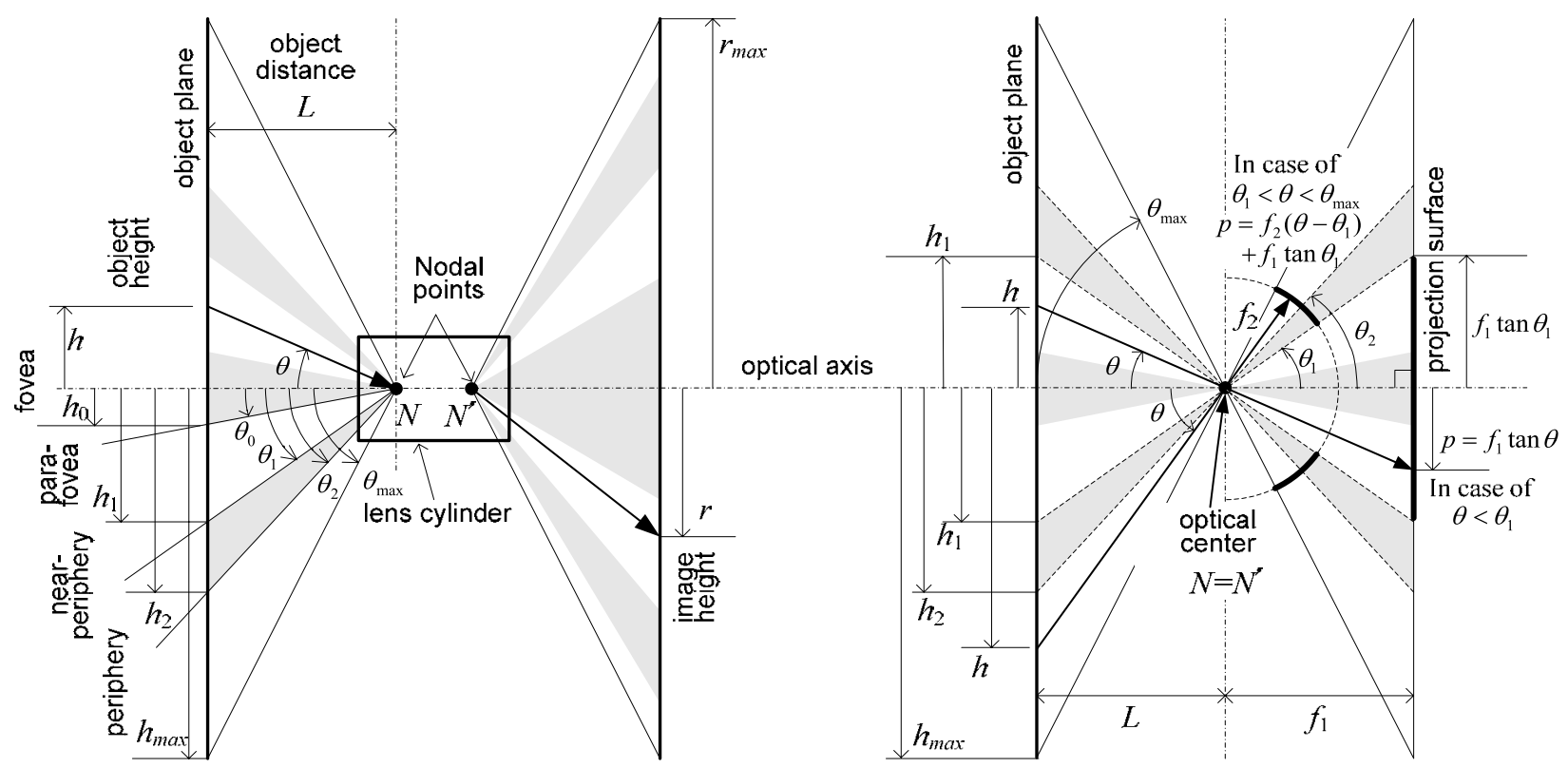

Fig. 2. Camera model based on combination of planer projection and spherical projection

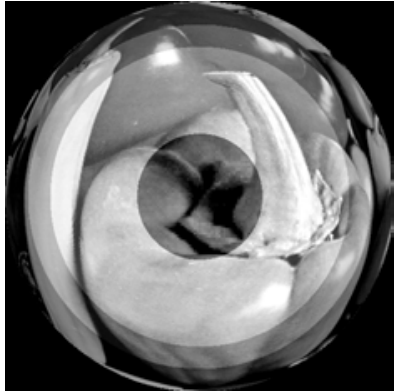

(a) AdWAF image

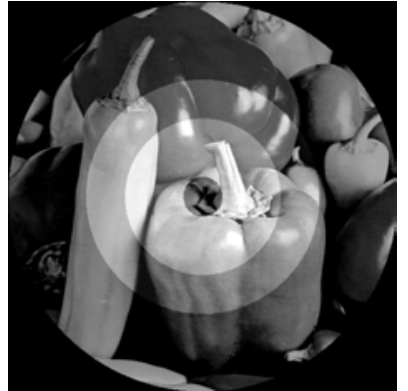

(b) linear-coordinate image

Fig. 3. Comparison of AdWAF model image and linearcoordinate PHC image

model (in condition that boundaries of the FOV, $\theta_{0}, \theta_{1}, \theta_{2}$ and $\theta_{\max }$ are $9.826^{\circ}, 19.107^{\circ}, 34.715^{\circ}$ and $60^{\circ}$ ), respectively. The intensity is changed in order to see each boundary easily: darker areas in the central FOV and the peripheral FOV are the fovea and the periphery, respectively, a brighter area surrounding the fovea is the para-fovea, and the original intensity area surrounding the para-fovea is the near-periphery.

Figure 4 shows the image height $r$, magnifications $d r / d h$ and $r / h$ in the radial and tangential directions of the AdWAF model, versus the object height $h$. The $h_{\max }$ and $r_{\max }$ are normalized to 1 (when $\theta_{\max }=60^{\circ}$ ) in order to compare other types of lens, that is, a log-polar (LP) lens, a fish eye (FE) lens, the PHC lens and the former WAF lens. In this simulation, the boundaries of the FOV, that is, $h_{0}, h_{1}$ and $h_{2}$, are $0.1\left(\theta_{0}=9.826^{\circ}\right), 0.4\left(\theta_{1}=19.107^{\circ}\right)$, and $0.6\left(\theta_{2}=34.715^{\circ}\right)$, respectively. Each type of lens is defined in the following. LP lens:

if $0 \leq \theta \leq \theta_{0}$ (fovea),

$$
r=r_{\max } f_{l p} \tan \theta
$$

else if $\theta_{0} \leq \theta \leq \theta_{\max }$ (periphery),

$$
r=r_{\max }\left\{\log _{a_{l p}}\left(f_{l p} \tan \theta\right)+d_{l p}\right\} .
$$

where $d_{l p}$ is denoted as

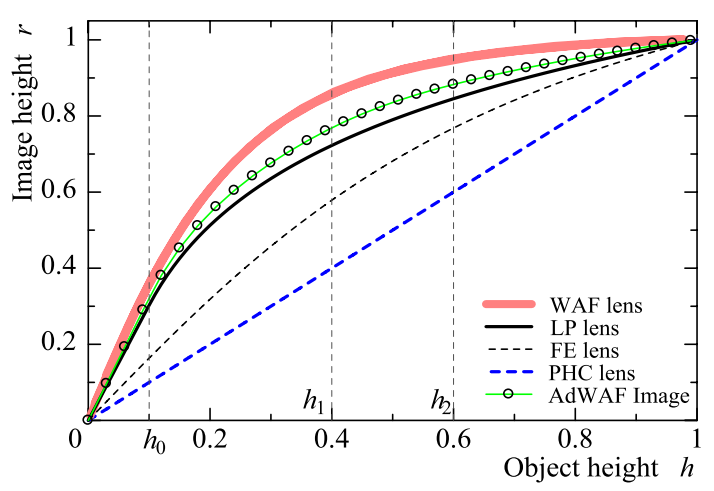

(a) image height $r$

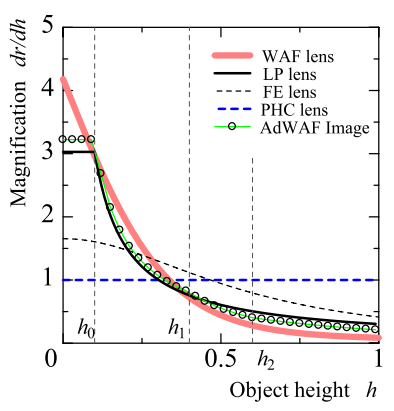

(b) magnification (in the radial)

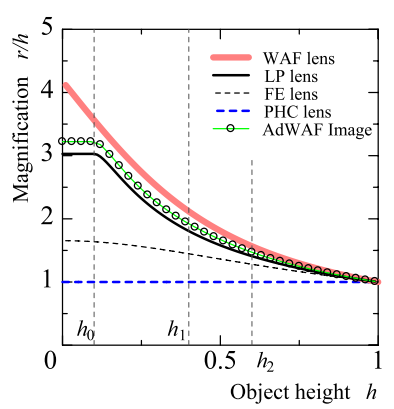

(c) magnification (in tangential)
Fig. 4. Comparison of AdWAF model and other types of lens

$$
d_{l p}=f_{l p} \tan \theta_{0}-\log _{a_{l p}}\left(f_{l p} \tan \theta_{0}\right),
$$

a focal length $f_{l p}$ is denoted as

$$
f_{l p}=\frac{1}{\left\{1+\log \left(\tan \theta_{\max } / \tan \theta_{0}\right)\right\} \tan \theta_{0}},
$$

a basis $a_{l p}$ is denoted as

$$
a_{l p}=\exp \left(\frac{1}{f_{l p} \tan \theta_{0}}\right),
$$

such that eqs. (14) and (15) are continuous at $\theta=\theta_{0}$, and 
their first derivations are also continuous. Note the LP lens is equivalent with the AdWAF model when $\theta_{1}=\theta_{2}=\theta_{\max }$, $c_{0}=c_{1}=1$ and $c_{2}=c_{3}=0$. Its fovea and 'periphery' correspond to the fovea and para-fovea of the AdWAF model, respectively.

FE lens:

$$
r=\frac{r_{\max }}{\theta_{\max }} \theta \quad\left(0 \leq \theta \leq \theta_{\max }\right),
$$

PHC lens:

$$
r=\frac{r_{\max }}{\tan \theta_{\max }} \tan \theta \quad\left(0 \leq \theta \leq \theta_{\max }\right), \cdots
$$

The former WAF lens:

$$
r=r_{\max }\left(a_{0} \theta^{3}+a_{1} \theta^{2}+a_{2} \theta\right) \quad\left(0 \leq \theta \leq \theta_{\max }\right) . \cdots
$$

A bold solid line shows the former WAF lens ${ }^{(2)}$. The distribution of its image height and magnification is characterized by the design concept of the WAF lens, i.e., achieving a wide FOV and high resolution in the central FOV. Its magnification in the radial direction is much higher than the PHC lens (a bold broken line) and the FE lens (a fine broken line) in small incident angles. On the other hand, it is lower in large incident angles. This figure shows the AdWAF model (a fine solid line with circle) can acquire higher magnification in the fovea $0 \leq h \leq h_{0}$ (that is, $0 \leq \theta \leq \theta_{0}$ ) than the LP lens (a solid line), in case of the same FOV. The scale modification factor $c_{i}$ is applicable for adjusting the image height of the AdWAF image in order to make its magnification in the fovea be equal to that of the LP lens. If $c_{0}=c_{1}=c_{2}=c_{3}=0.93$, the modified magnification is nearly equal to that of the LP lens in the fovea in case of Fig. 4(b). This means the AdWAF model can reduce the number of pixels by $13.5 \%$ in the whole of image comparing with the LP lens.

3.2 Target Specification The former WAF lens was designed and produced as being attached with commerciallyavailable C-mount CCD and CMOS cameras having a 1/2 inch imaging chip. This lens has a $32 \mathrm{~mm}$ length along the optical axis and a $16 \mathrm{~mm}$ diameter. The maximum image height corresponding to an incident angle 60 degrees is $2.4 \mathrm{~mm}$. In those days when the former WAF lens was produced, it was sufficiently-compact. But right now we need a more compact WAF lens because the imaging chip technology has been advanced rapidly, i.e., a photosensitive element has got smaller time by time and the number of pixels in the imaging chip has increased drastically. A camera system itself also has got smaller as a board lens camera and an embedded USB camera (i.e., used for a mobile phone, and notebook PC). This is a motivation by which the more compact WAF lens, i.e., Micro WAF lens, is designed for a board lens camera using the AdWAF model. Target specification is in the following items and Table 1.

(1) Size: A length should be smaller than $15 \mathrm{~mm}$, and the maximum lens diameter should be smaller than $8 \mathrm{~mm}$ in order to attach with a board camera.

(2) A percentage of the central FOV, i.e., fovea within an incident angle of $2.479^{\circ}$, should be approximately $10 \%$ of the entire FOV, i.e., an area within a circle corresponding to the maximum incident angle of $60^{\circ}$, in order to improve resolution of the central FOV. Concretely we set $\theta_{0}, \theta_{1}$ and $\theta_{2}$ as $2.479^{\circ}, 14.564^{\circ}$ and
Table 1. Target Specification

\begin{tabular}{|c|c|c|}
\hline & The former WAF lens & Micro WAF lens \\
\hline Maximum image angle & $120^{\circ}$ & $120^{\circ}$ \\
\hline $\begin{array}{c}\text { Maximum image height } \\
\text { (Imaging chip size })\end{array}$ & $\begin{array}{c}2.4 \mathrm{~mm} \\
(1 / 2 \text { inch imaging chip })\end{array}$ & $\begin{array}{c}1.5 \mathrm{~mm} \\
(1 / 3 \text { inch imaging chip })\end{array}$ \\
\hline Fovea region & $\begin{array}{c}10 \% \text { within incident angle of } \\
10^{\circ}\end{array}$ & $\begin{array}{c}10 \% \text { within incident angle of } \\
2.5^{\circ}\end{array}$ \\
\hline Camera as a target & Industrial C-mount camera & Board lens camera \\
\hline Lens size & $\phi 16 \mathrm{~mm} \times 32 \mathrm{~mm}$ & Smaller than $\phi 8 \mathrm{~mm} \times 15 \mathrm{~mm}$ \\
\hline
\end{tabular}

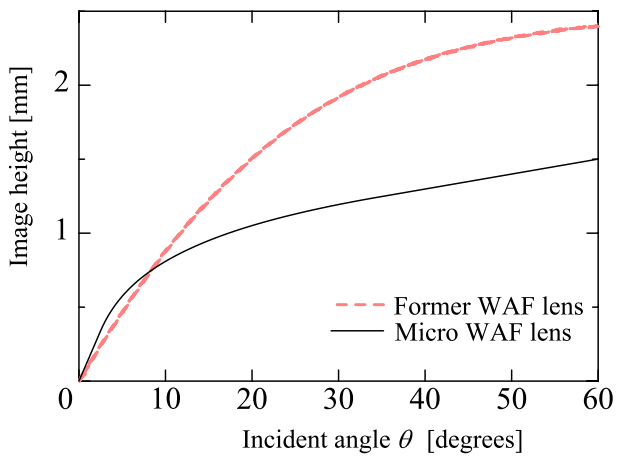

(a) actual image height [mm]

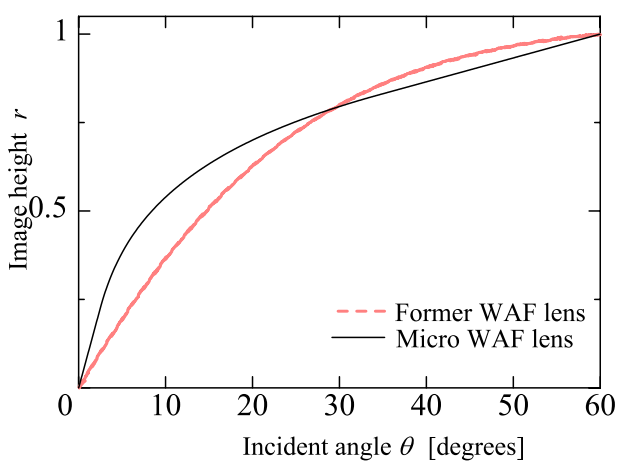

(b) normalized image height $r$

Fig. 5. Micro WAF lens and Former WAF lens

$34.715^{\circ}$, respectively.

(3) The lens is designed as being attached with $1 / 3$ inch imaging chip, i.e., the maximum image height corresponding to $60^{\circ}$ is determined as $1.5 \mathrm{~mm}$. However, we do not pay attention only to a projected image size but also to light illumination which enters each photosensitive element. We need to take into account a smaller opening size of the photosensitive element caused by enormous increment of the number of pixels in the imaging chip.

Figure 5(a) shows the image heights of the former WAF lens (a broken line) and Micro WAF lens (a solid line) versus an incident angle $\theta$. This image height function of Micro WAF lens is used as a part of a merit function of the optical CAD software later. Figure 5(b) compares the image heights of both WAF lenses, in which the maximum image height is normalized to 1 . It is noted that Micro WAF lens has a more magnified fovea region than the former WAF lens, because a gradient of the image height curve in Fig. 5 means magnification of the lens. Figure 6 compares simulated images among (a) a sample image, (b) the former WAF lens image, (c) Micro 


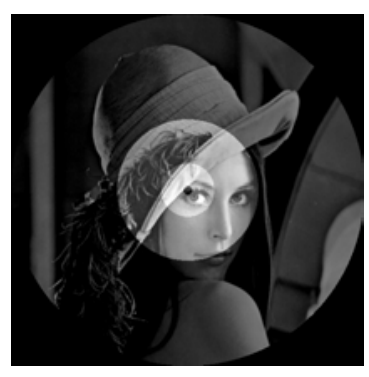

(a) Sample image

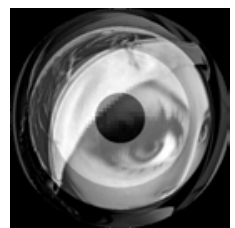

(c) Micro WAF lens (actual size)

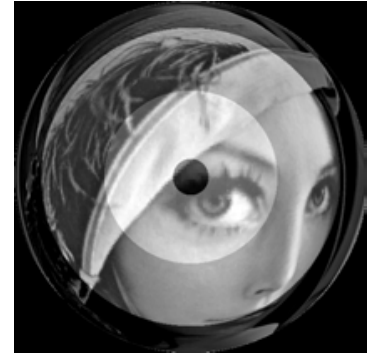

(b) Former WAF lens

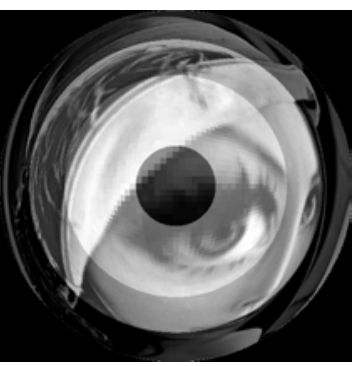

(d) Micro WAF lens
Fig. 6. Comparison of WAF lens images

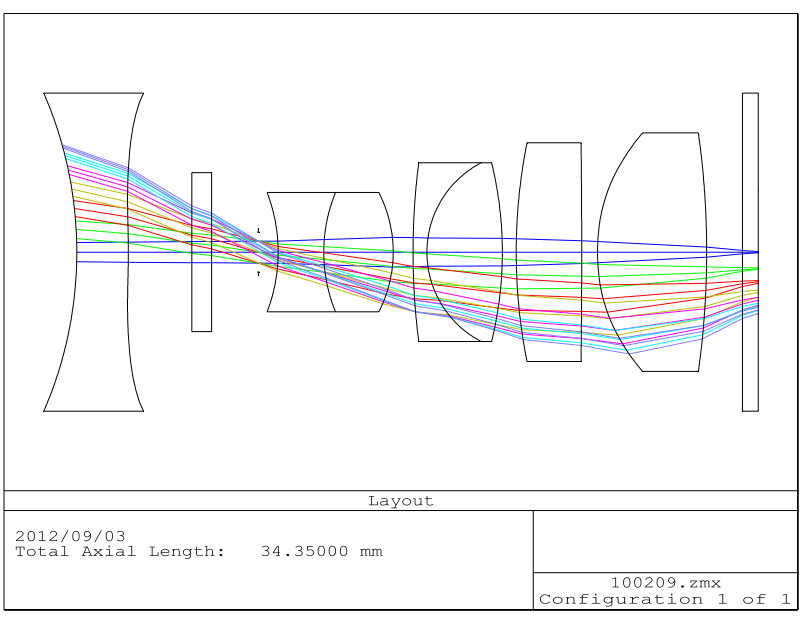

(a) compound system of the former WAF lens

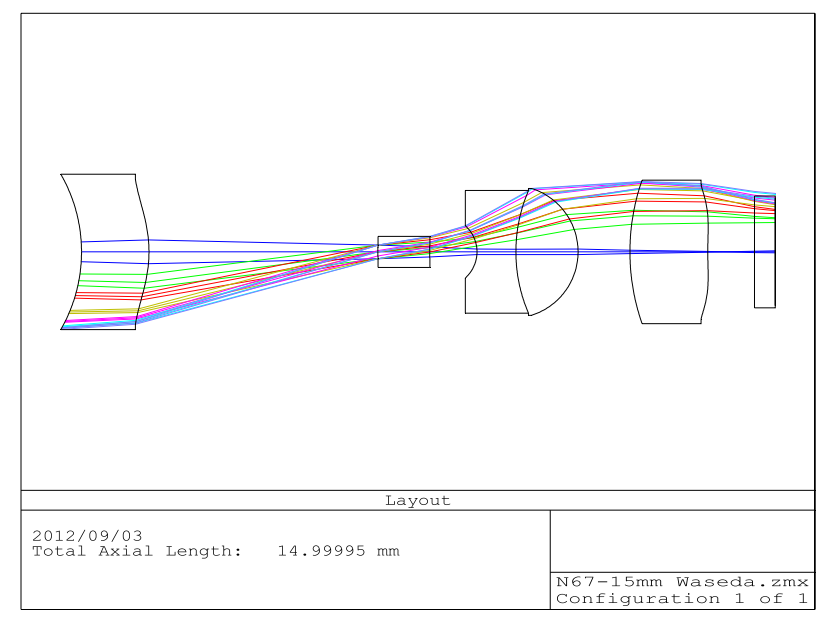

(b) compound system of Micro WAF lens

Fig. 7. Comparison of compound systems

WAF lens image (original), and (d) Micro WAF lens image where the maximum image height is given as being the same as the former WAF lens. Also from this figure, we note that

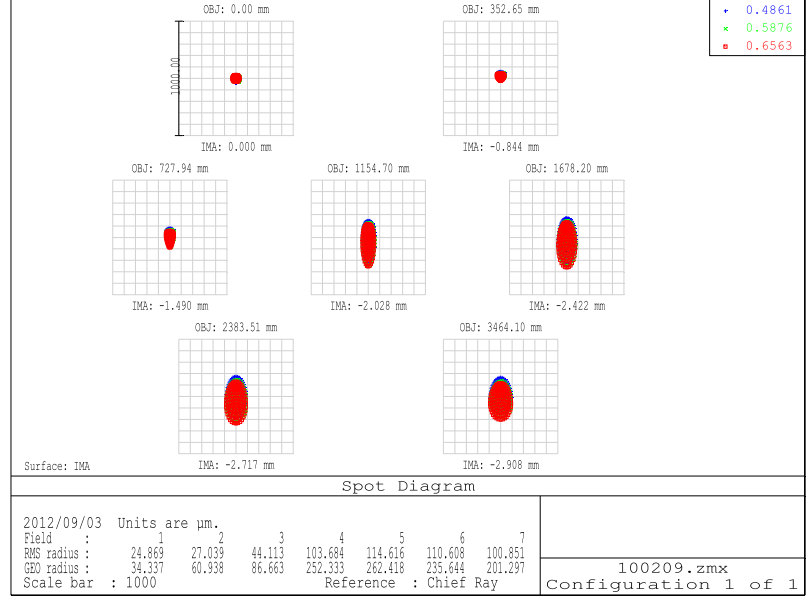

(a) spot diagram of the former WAF lens

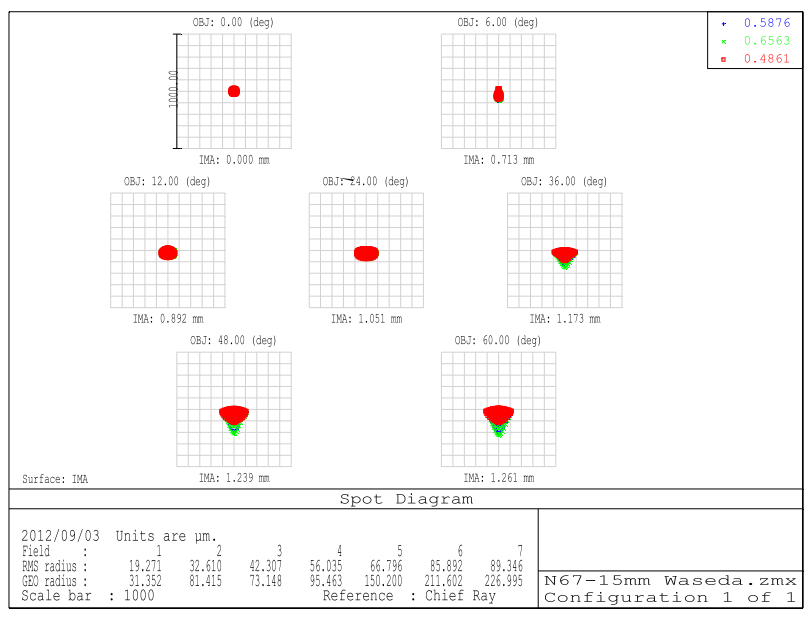

(b) spot diagram of Micro WAF lens

Fig. 8. Comparison of spot diagram

Micro WAF lens has the more magnified fovea region than the former WAF lens in spite of its smaller entire FOV.

3.3 Lens Design by Simulation Micro WAF lens is designed based on the Damping Least Square (DLS) method using ZEMAX-EE (Radiant ZEMAX LLC) as optics design software. We refer to a compound system of the former WAF lens composed of 7 lenses (Fig. 7(a)). In order to make the total length of a designed lens be smaller, we reduce the number of lenses in the compound system from 7 to 5 (Fig. 7(b)). In Figs. 7(a) and 7(b), the most right optical parts are a cover glass of the imaging chip. In Fig. 7(a), the 2nd and 3rd parts from the left are an IR cut filter and a fixed diaphragm, respectively. These compound systems do not count these parts as a lens. Although the former WAF lens used 2 pairs of doublet, Micro WAF lens reduces the number of doublet into 1 pair by using a concave lens having a large curvature (on the 5th surface from the left) and a planar glass having a comparatively-large thickness (the 2nd lens from the left in Fig. 7(b)). This is a significant point for minimizing the WAF lens successfully. Figure 8 compares a spot diagram between (a) the former WAF lens and (b) Micro WAF lens. In spite of decreasing the number of the lenses from 7 to 5 and being minimized, Micro WAF lens improves chromatic aberration and astigmatism in the radial direction around the image center (an intersection point between the optical axis and an 


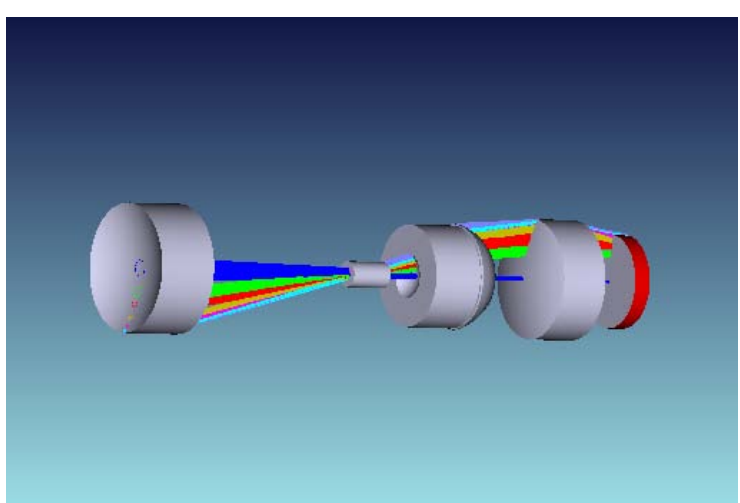

Fig. 9. Shade model of Micro WAF lens
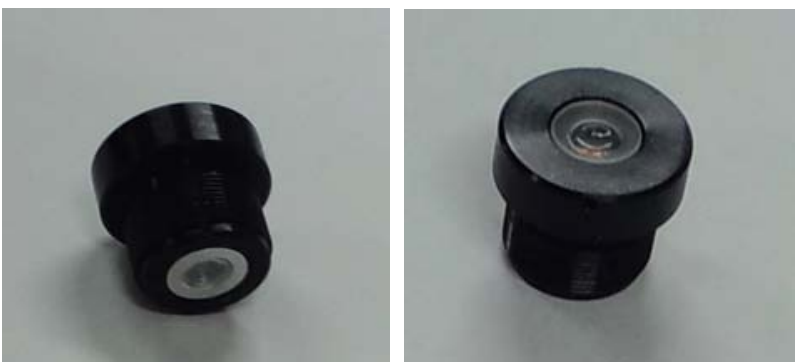

Fig. 10. Pictures of a prototype of Micro WAF lens

image plane) on the image plane. Figure 9 shows a designed shade model of Micro WAF lens.

3.4 Production of a Prototype Figure 10 shows pictures of a prototype of Micro WAF lens we have produced. A size of the lens compound system is a $12 \mathrm{~mm}$ length and a $3 \mathrm{~mm}$ diameter (when not including a lens cylinder). Currently, the author inspects an optical performance of this prototype.

\section{Conclusion}

4.1 Results In this paper, the authors developed a micro WAF lens, minimized for attaching with a board lens camera having a $1 / 3$ inch imaging chip. We applied the AdWAF model for the lens design in order to realize the fovea region having a higher resolution than that in the case of the former WAF lens and the para-fovea region having a rotationand scale-invariant property. As a result, despite the fact that the designed Micro WAF lens has a much smaller size, we achieved a better optical performance in its computer simulation.

4.2 Future Works First, an inspection process of the produced Micro WAF lens needs to be completed by comparing its performance with simulation results. Next, we produce a small view direction control machine suitable for the board lens camera where Micro WAF lens is attached in order to realize a smart visual information collection from an environment. In addition, we are planning to minimize the WAF lens furthermore to a tiny lens size applicable for an embedded USB camera.

\section{Acknowledgment}

This research is partially supported by JKA through its Promotion funds from Keirin RACE (23-88). The author would like sincerely to thank Kyoji Nariai, Ph.D., an emeritus professor at National Astronomical Observatory of Japan for minimizing the lens in the design process, and appreciate Joel W. Burdick, a professor at California Institute of Technology for a theoretical verification of the AdWAF model.

\section{References}

( 1 ) S. Shimizu: "Multi-Functional Application of Wide Angle Foveated Vision Sensor in Mobile Robot Navigation", Journal of Robotics and Mechatronics, Vol.14, No.4, pp.382-389 (2002-8)

( 2 ) S. Shimizu, et al.: "Vision Sensor with Wide Angle and High Distortion lens", Video proceedings of IEEE International Conference on Robotics and Automation, Visual Sensing 3 (1995-5)

( 3 ) Y. Kuniyoshi, N. Kita, K. Sugimoto, et al.: "A Foveated Wide Angle Lens for Active Vision", Proc. of IEEE International Conference on Robotics and Automation, pp.2982-2988 (1995-5)

( 4 ) E.L. Schwartz: "Spatial mapping in the primate sensory projection: Analytic structure and relevance to perception", Biological Cybernetics, Vol.25, No.4, pp.181-194 (1977-12)

( 5 ) G. Sandini and V. Tagliasco: "An anthropomorphic retina-like structure for scene analysis", Computer Graphics and Image Processing, Vol.14, No.4, pp.365-372 (1980-12)

( 6 ) F. Berton, G. Sandini, and G. Metta: "Anthropomorphic Visual Sensors", Encyclopedia of Sensors, Edited by C.A. Grimes, E.C. Dickey and M.V. Pishko, Vol.10, pp.1-16 (2005)

( 7 ) J. Van der Spiegel, G. Kreider, C. Claeys, I. Debusschere, G. Sandini, P. Dario, and et al.: "A foveated retina-like sensor using CCD technology", Analog VLSI Implementations of Neural Networks. Kluwer, C. Mead and M. Ismail, Boston (1989)

( 8 ) R. Wodnicki, G.W. Roberts, and M.D. Levine: "A foveated image sensor in standard CMOS technology", In Custom Integrated Circuits Conference, Santa Clara, California (1995-5)

( 9 ) S. Shimizu, H. Jiang, and J.W. Burdick: "Image Extraction by Wide Angle Foveated Lens for Overt-Attention", Proc. of IEEE International Conference on Robotics and Automation, pp.3437-3442 (2006-5)

(10) S. Shimizu: "Wide-Angle Foveation for All-Purpose Use", IEEE/ASME Transactions on Mechatronics, Vol.13, No.5, pp.587-597 (2008)

(11) Caltech, Inventor: S. Shimizu: "Imaging Model and Apparatus", U.S. Patent, US8,094,965B2 (2012-1)

(12) Caltech, Inventors: S. Shimizu, and J.W. Burdick: "Image Processor", U.S. Patent, US8,094,169B2 (2012-1)

Sota Shimizu (Member) was born in Nagoya, Japan, in 1970. He re-

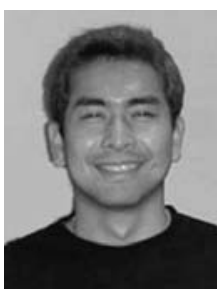
ceived the Ph.D. degree in electronic-mechanical engineering from Nagoya University, Nagoya, in 1998. From 2000 to 2002, he was with Tokai University as an Assistant Professor. Since 2004, he has been with the California Institute of Technology, Pasadena. Currently, he is an Associate Professor with Interfaculty Initiative in Information Studies, The University of Tokyo. His current research interests include cognitive science, analysis of eye movement, bio-mimetic vision sensor and artificial eye.

Takumi Hashizume (Non-member) was born in Tajimi, Japan, in

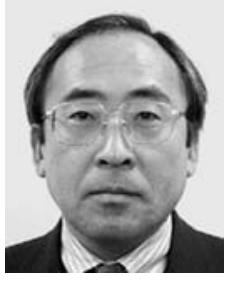
1951. He received the Ph.D degree in School of Science and Engineering, Waseda University. Since 1980, he has been with Waseda University. Currently, he is a Professor with Advanced Research Institute of Science and Engineering, Waseda University. His current research interests include geometrical and environmental measurement using a small unmanned aerial vehicle and Mobile Mapping System, automation, and high-efficiency production system. 